九州大学学術情報リポジトリ

Kyushu University Institutional Repository

Effects of capping layers on the photoelectrochemical property of silver nanoparticle-modified indium-tin-oxide electrode

\title{
Matsuoka, Ken-Ichi
}

Department of Materials Physics and Chemistry, Graduate School of Engineering, Kyushu University

\section{Tahara, Hironobu}

Department of Materials Physics and Chemistry, Graduate School of Engineering, Kyushu University

Akiyama, Tsuyoshi

Department of Applied Chemisty, Faculty of Engineering, Kyushu University | Department of Materials Physics and Chemistry, Graduate School of Engineering, Kyushu University

\section{Yamada, Sunao}

Department of Applied Chemisty, Faculty of Engineering, Kyushu University | Department of Materials Physics and Chemistry, Graduate School of Engineering, Kyushu University

http://hdl. handle. net/2324/25482

出版情報: Journal of Photochemistry and Photobiology A : Chemistry. 221 (2/3), pp. 239-243， 2011-06-25. Elsevier

バージョン:

権利関係: (C) 2011 Elsevier B.V. 
Effects of Capping Layers on the Photoelectrochemical Property of Silver Nanoparticle-modified Indium-Tin-Oxide Electrode

\author{
Ken-ichi Matsuoka ${ }^{1}$, Hironobu Tahara ${ }^{1}$, Tsuyoshi Akiyama ${ }^{1,2}$, Sunao Yamada ${ }^{* 1,2}$ \\ ${ }^{1}$ Department of Materials Physics and Chemistry, Graduate School of Engineering, Kyushu University \\ 744 Motooka, Nishi-ku, Fukuoka 819-0395, Japan \\ ${ }^{2}$ Department of Applied Chemisty, Faculty of Engineering, Kyushu University. \\ 744 Motooka, Nishi-ku, Fukuoka 819-0395, Japan
}

\begin{abstract}
Photoelectrochemical properties of indium-tin-oxide (ITO) substrate modified with silver nanoparticles (AgNPs) and effects of surface capping layer of AgNP were investigated in the water/acetonitrile $\left(\mathrm{H}_{2} \mathrm{O} / \mathrm{MeCN}\right)$ electrolyte solutions with the presence of oxygen as an electron acceptor. The modified ITO substrate showed photocurrent response mainly driven by the photoinduced electron transfer from ITO substrate to AgNPs. In addition, its photoelectrochemical property significantly depended on the solvent composition of electrolyte solution which was in correlation with surface status of AgNPs. Combination of photoelectrochemical measurements and spectroscopic analysis suggested that presence of considerable amount of $\mathrm{MeCN}$ in the electrolyte solution triggered partial displacement of capping citrate ions to activate photocurrent generation.
\end{abstract}




\section{Introduction}

Understanding of photoelectrochemical processes between semiconductor and metal nanoparticle (MNP) is one of the central issues in the range of photocatalytic and photovoltaic applications [1-5]. To date, photoelectrochemical properties of several semiconductor-MNP composite systems, such as $\mathrm{ZnO}-\mathrm{MNP}[1,2]$ and $\mathrm{TiO}_{2}-\mathrm{MNP}$ [3-5], have been investigated to evaluate their peculiar photoelectric interactions and potential utilities in related device performance.

Photoelectrochemical interaction between indium-tin-oxide (ITO) and MNP is a particular interest since ITO is one of the versatile semiconducting materials and offers highly conductive and transparent supports for the various MNP-based photoelectrochemical architectures [6-10]. In particular, our group recently reported that AuNP [6,7] and AgNP [8] arrays deposited on ITO substrate substantially enhanced photocurrent signals from attached organic dyes due to the electric field generated by the localized surface plasmon (LSP) excitation.

In such systems, LSP-assisted excitation of organic dyes have been the main focus, but other potential factors for the overall photoelecrochemical outputs, such as photoinduced interactions between the substrate and NMPs, have not been carefully examined. Along with this line, clarification of the photoelectric interaction between ITO substrate and MNPs will offer an additional basis for the development of photoactive nanostructures to utilize unique optical and electrochemical properties of MNPs. These circumstances encouraged us to investigate the photoelectrochemical properties of ITO substrates modified with AuNP and AgNP arrays $[11,12]$. However, clarification of such photoelectrochemical systems has not been accomplished yet.

We report herein the preliminary investigation of the photoelectrochemical property involving AgNP and ITO substrate with an emphasis on the effect of capping layers of AgNP. The emphasis was made as considering the fact that capping layers on MNPs regulate wide range of physical aspects such as optical [13], conductive [14], catalytic [15], electrochemical [16], and photoelectrochemical [17] properties. In this study, photoinduced electron transfer 
in ITO-AgNP system and deep correlation between the surface status of AgNPs and the photoelectrochemical performation of AgNP-deposited ITO substrate were evaluated.

\section{Experimental}

\subsection{Materials}

Silver nitrate $\left(\mathrm{AgNO}_{3}, 99.5 \%\right.$, Wako), trisodium citrate dehydrate (99\%, Wako), sodium tetrahydroborate $\left(\mathrm{NaBH}_{4}, 97 \%\right.$, Kishida), sodium chloride (NaCl, $99.5 \%$, Wako), poly(ethyleneimine) $\left(\mathrm{PEI}, M_{\mathrm{w}}=50,000-100,000\right.$, Wako), sodium perchlorate $\left(\mathrm{NaClO}_{4}, 95 \%\right.$, Wako), acetonitrile (MeCN, $99.5 \%$, Wako), 1-octadecanethiol (ODT, $\geqq 90 \%$, wako), and hydroxyethanethiol (95\%,Wako) were used as received. Pure water $(18 \mathrm{M} \Omega \cdot \mathrm{cm})$ was obtained from Mill-Q Academic A-10 system (Millipore).

\subsection{Preparation of AgNPs}

Citrate-capped AgNPs were synthesized by the modified method of Wang et al. [18]. Briefly, an aqueous solution of $0.5 \mathrm{mM} \mathrm{AgNO}_{3}(100 \mathrm{ml})$ was added into the equivalent volume of aqueous solution containing $2 \mathrm{mM}$ of $\mathrm{NaBH}_{4}$ and $0.13 \mathrm{mM}$ of trisodium citrate under the cooling in ice-bath, forming a yellowish colloidal solution of AgNPs. The colloidal solution was heated to $70{ }^{\circ} \mathrm{C}$ to decompose excess $\mathrm{NaBH}_{4}$, and then cooled to room temperature. The average diameter of resultant AgNPs was evaluated to be $13.5 \pm 3.3 \mathrm{~nm}$ from the transmission electron microscope (TEM) images (Fig. S1).

\subsection{Fabrication of AgNP-modified ITO substrate}

The ozone-cleaned ITO substrate (200 nm thickness of ITO film deposited on the glass substrate, Geomatec co. ltd., $10 \Omega$ /square) was immersed into an aqueous solution of PEI (45 $\mathrm{mg} / \mathrm{ml}$ ) containing $0.2 \mathrm{M} \mathrm{NaCl}$ for $10 \mathrm{~min}$ at $30{ }^{\circ} \mathrm{C}$. After withdrawal, the substrate was 
washed with $\mathrm{H}_{2} \mathrm{O}$ under sonication. The resultant PEI-coated ITO substrate is denoted as ITO/PEI. The AgNP-modified ITO substrate, denoted as ITO/PEI/AgNP, was obtained by soaking ITO/PEI into the colloidal solution of AgNPs for $12 \mathrm{~h}$ at room temperature, followed by rinsing with water.

\subsection{Measurements}

UV-vis spectral measurements were carried out by JASCO V-670 spectrophotometer. Transmittance-reflectance spectral measurements were carried out by Shimazu UV-3150 spectrophotometer. Scanning electron microscope (SEM) images were taken on Hitachi S-5000 electron microscope with an acceleration voltage of $10 \mathrm{kV}$. TEM images were taken on JEOL JEM-2010 electron microscope with an acceleration voltage of $120 \mathrm{kV}$. Quartz crystal microbalance (QCM) measurements were performed with ALS Model 420 Electrochemical analyzer. Gold-covered QCM plates (8 $\mathrm{MHz}$ ) was used for QCM measurements in order to estimate the thickness of PEI film. Namely, the QCM plate was immersed in an ethanol solution of $1 \mathrm{mM}$ hydroxyethanethiol for one day to implant hydroxyl groups on the surface of QCM plate prior to PEI coating [12]. Photocurrent measurements of ITO/PEI/AgNP (working) were carried out by the three-electrode photoelectrochemical cell using a $\mathrm{Ag} / \mathrm{AgCl}$ (sat. $\mathrm{KCl}$ ) electrode (reference) and a platinum coil (counter). Oxygen bubbling was carried out for $60 \mathrm{~min}$ prior to the measurements. Monochromatic light from a Xe lamp irradiated the modified ITO substrate (irradiation area $: 0.28 \mathrm{~cm}^{2}$ ) from the back side of the substrate, where AgNPs were not doposited, and the resultant photocurrents were measured with Huso HECS-318C potentiostat. The irradiation power of the monochromatic right was determined by OPHIR power/energy meter NOVA.

\section{Results and Discussion}

\subsection{Characterization of ITO/PEI/AgNP substrate}


Characterization of ITO/PEI/AgNP was carried out by UV-vis, SEM, and QCM measurements. Fig. 1b shows a typical UV-vis spectrum of ITO/PEI/AgNP. It exhibits broad bands around 420 and $580 \mathrm{~nm}$, respectively. The former is mainly attributable to the plasmon band of isolated AgNPs, while the latter due to interparticle plasmon coupling of aggregated AgNPs. SEM image of ITO/PEI/AgNP (Fig. 1a) shows randomly deposited AgNP array (bright spots) on ITO/PEI. Presence of both isolated and aggregated AgNP was confirmed. The surface coverage of AgNP was estimated to be $\sim 1.6 \times 10^{11}$ particles per 1 $\mathrm{cm}^{2}$ from the SEM image, which corresponds to $22 \%$ of surface coverage based on the average diameter of AgNP. Thickness of PEI film was estimated from QCM measurements. Frequency decrease of $177 \pm 7 \mathrm{~Hz}$ was obtained upon PEI coating. This value corresponds to ca. $5.0 \pm 0.2 \mathrm{~nm}$ thickness of PEI film by assuming the reported density $\left(1.2 \mathrm{~g} \mathrm{~cm}^{-3}\right)$ [19].

\subsection{Solvent-dependent stability and surface status of AgNP}

Stability of AgNP array electrostatically deposited on ITO substrates was investigated in various $\mathrm{H}_{2} \mathrm{O} / \mathrm{MeCN}$ mixed solvents as a preparative step for photoelectrochemical measurements. Fig. 2 shows the time dependence of UV-vis spectral change of ITO/PEI/AgNP in $\mathrm{H}_{2} \mathrm{O} / \mathrm{MeCN}$ solutions with various volume ratios $\left(\mathrm{H}_{2} \mathrm{O} / \mathrm{MeCN}=10 / 0-\right.$ 0/10; v/v) under aerated condition. As shown in Figs. 2a and b, no significant spectral changes were observed within the experimental period $(120 \mathrm{~min})$ when the volume fraction of MeCN was lower $\left(\mathrm{H}_{2} \mathrm{O} / \mathrm{MeCN}=10 / 0,9 / 1\right)$. Slight red-shift of spectrum is probably due to the local change of particle aggregation. In contrast, gradual decrease of plasmon band of AgNPs was observed when the volume fraction of MeCN was $30-50 \%$ (Figs. 2c and d). In addition, such spectral change became significant when the volume fraction of $\mathrm{MeCN}$ was larger than $70 \%$ (Figs. 2e and f). The degradation of plasmon band is attributable to the oxidation of AgNPs by oxygen. This was confirmed by the fact that purging the MeCN $\left(\mathrm{H}_{2} \mathrm{O} / \mathrm{MeCN}=0 / 10\right)$ solution with nitrogen gas $(15 \mathrm{~min})$ prior to the $\mathrm{UV}$-vis measurements effectively reduced the degradation of plasmon band (Fig. $2 \mathrm{~g}$ ) in comparison to the case under aerated condition (Fig. 2f). 
Possible reason for the solvent-dependent oxidation of AgNP is partial displacement of citrate ions by $\mathrm{MeCN}$ present in the solution. It has been reported that $\mathrm{MeCN}$ molecules reversibly adsorb on the Ag surface through the interaction of nitrile group [20,21], where the activation energy of $\mathrm{MeCN}$ desorption, for example, was reported to be $10 \mathrm{kcal} / \mathrm{mol}$ at the (110) surface [20]. Adsorption of $\mathrm{MeCN}$ accompanies the removal of capping citrate ions. Thus, it is plausible that oxidation of AgNPs proceeds at the deprotected areas. Displacement of citrate ions must be accelerated by increasing the volume fraction of $\mathrm{MeCN}$ as can be recognized from Figs. 2a-f.

This assumption is further supported by the fact that the spectral degradation was remarkably suppressed by adding capping reagent $(0.1 \mathrm{mM}$ trisodium citrate $)$ in the $\mathrm{H}_{2} \mathrm{O} / \mathrm{MeCN}=3 / 7$ solution (Fig. 2h), as compared with the case without trisodium citrate (Fig. 2e). In general, citrate ion is known as a capping reagent of metal colloids as well as a thermal reducing reagent [22]. However, reduction of $\mathrm{Ag}^{+}$with citrate ion hardly proceeds at room temperature. Thus, these results suggest that the oxidation of AgNPs was mainly suppressed by securing the AgNP surface with citrate ions.

\subsection{Photoelectrochemical property of ITO/PEI/AgNP and effect of surface status of AgNP}

The photoelectrochemical property of ITO/PEI/AgNP was investigated in the $0.1 \mathrm{M}$ $\mathrm{NaClO}_{4}$ electrolyte solution using $\mathrm{H}_{2} \mathrm{O} / \mathrm{MeCN}$ mixed solvents $\left(\mathrm{H}_{2} \mathrm{O} / \mathrm{MeCN}=10 / 0,9 / 1,7 / 3\right.$, 5/5; v/v) under oxygen-saturated condition. Photocurrent action spectra, except for ITO/PEI, were obtained by averaging the results of three independent samples.

Fig. 3 shows the relationship between photocurrent action spectra of the samples and electrolyte solution conditions. The photocurrents from ITO/PEI/AgNP were negligibly small when the volume fraction of $\mathrm{MeCN}$ was lower $\left(\mathrm{H}_{2} \mathrm{O} / \mathrm{MeCN}=10 / 0,9 / 1\right)$. On the contrary, distinct cathordic photocurrents were observed when the volume fraction was relatively higher $\left(\mathrm{H}_{2} \mathrm{O} / \mathrm{MeCN}=7 / 3,5 / 5\right)$. Remarkable photocurrent was observed up to around $450 \mathrm{~nm}$ with a small peak at $380 \mathrm{~nm}$. A control experiment without AgNP (ITO/PEI) in $\mathrm{H}_{2} \mathrm{O} / \mathrm{MeCN}=7 / 3$ showed negligible photocurrent response. It is notable that the sudden 
increase of the cathordic photocurrent signal and the degradation of the plasmon band of AgNP (partial deprotection of citrate ions as described in the previous section) were observed at the same volume fraction of $\mathrm{MeCN}$ (30 vol.\%). In addition, our previous studies showed that PEI film had no remarkable effects on the photoelectrochemical properties of the similar systems $[7,8,11,12]$. Therefore, it is strongly suggested that the photocurrent generation process involves partially deprotected AgNPs .

As shown in Fig. 3, photocurrent profiles of ITO/PEI/AgNP do not match the plasmon band of AgNPs. Therefore, we speculated that excitation of the ITO substrate is the major contribution of photocurrent. To verify this, the absorption profile of the ITO substrate was evaluated by transmittance-reflectance measurements. Absorption coefficient $\alpha(h v)$ of ITO was estimated by the following equation [23]

$$
\alpha(h v)=\frac{1}{l} \ln \frac{1-R(h v)}{T(h v)}
$$

where $R(\lambda)$ and $T(\lambda)$ correspond to reflectance and transmittance of ITO, respectively. $\quad l$ is the thickness of ITO film (200 nm in this study). The absorption coefficient can be described by the relation for parabolic bands as follows [24]

$$
(\alpha h v)^{N} \propto h v-E_{g}
$$

where $h v$ represents the photon energy, $E_{g}$ the optical band gap, and $N$ depends on the type of electron transition: i.e. direct transition corresponds to $N=2$ and indirect transition correspond to $N=1 / 2$. As shown in Fig. 4a, we found a linear trend at higher photon energy region with an onset around $3.7 \mathrm{eV}$ corresponding to the direct band gap energy under the condition of $N=2$. On the other hand, the indirect band gap energy of around $2.6 \mathrm{eV}$ was also obtained in a similar manner from the analysis at $N=1 / 2$. These values are in agreement within the reported values $[23,24]$.

Fig. $4 \mathrm{~b}$ shows the absorption coefficient of the ITO substrate. In the present study, photocurrent generation becomes significant at the wavelength shorter than $450 \mathrm{~nm}(2.75 \mathrm{eV}$, see Fig. 3), which is fairly correlated with the obtained indirect band gap energy $2.6 \mathrm{eV}$. Absorption at longer wavelength region $(>530 \mathrm{~nm})$ is ascribed to the plasma oscillation. In addition, ITO substrate has an absorption peak around $380 \mathrm{~nm}$. This value matches well 
with the observed photocurrent peak at $380 \mathrm{~nm}$ (Fig. 3). These results imply that excitation of ITO substrate is the major contribution to the photocurrent generation.

It was expected that excited electrons in ITO substrate are first transferred to the attached AgNPs. Then, stored electrons in AgNPs participate in the reduction of oxygen to generate cathordic photocurrent. Solvent-dependent characteristics of photocurrent generation suggested that oxygen was dominantly reduced at the deprotected surface of AgNPs to generate cathordic photocurrent. It should be noted, however, that observed photocurrent profile and absorption profile of ITO substrate do not overlap completely. Photocurrent profile of ITO/PEI/AgNP showed shoulders around $410 \mathrm{~nm}$ while no such shoulder can be recognized in the absorption profile of ITO substrate. Since AgNP exhibited strong absorption close to the photocurrent shoulder with maximum around $420 \mathrm{~nm}$, plasmon-excitation of AgNPs could also participate in the reduction of oxygen. Such phenomena have been observed in roughed Ag substrates under surface plasmon excitation [25]. Further studies are necessary to address this issue.

We also investigated the effect of the surface status of AgNPs on photocurrent generation by two experiments. First, photocurrent measurement was carried in the presence of $0.1 \mathrm{M}$ trisodium citrate in the solution $\left(\mathrm{H}_{2} \mathrm{O} / \mathrm{MeCN}=3 / 7\right)$. Second, AgNPs were modified with ODT by soaking ITO/PEI/AgNP in the ethanol solution containing $1 \mathrm{mM}$ of ODT for one day. Then, the photocurrents of the ODT-modified sample were compared in the same electrolyte solution without trisodium citrate. Results of photocurrent measurements are shown in Fig. 5. Significant decrease of photocurrent was also observed in both cases. As shown in Fig. $2 \mathrm{~h}$, presence of enough citrate ions in the surrounding medium effectively protects AgNP surface from oxygen. This is likely to inhibit the reduction process of oxygen upon the photoinduced electron injection from ITO to AgNPs. Surface-immobilized ODT layer also suppressed the reduction of oxygen at AgNP surface. Such barrier effect toward oxygen has been reported by Tatsuma et al. in $\mathrm{AgNP} / \mathrm{TiO}_{2}$ composite system [17]. These results further suggest the correlation between the surface status of AgNP and photoelectrochemcal property of ITO/PEI/AgNP in the present experimental condition. 


\section{Conclusion}

In summary, photoelectrochemical interaction between ITO substrate and AgNPs and the effect of surface status of AgNP on the photocurrent generation were investigated. Presence of photoinduced electron injection from the excited ITO substrate to AgNPs was strongly suggested by the analysis of the photocurrent profiles. Citrate ions and ODT capping layers on the AgNP surface exhibited barrier effect against oxygen. Meanwhile, MeCN removes some of the capping citrate ions from the AgNP surface, giving the photoelectrochemical activity to the system.

It was demonstrated that electrochemically negatively-biased AuNP suppressed electron transfer process between AuNP and attached organic dye by repelling the electrons from excited dye [26]. Along with this line, photoinduced electron injection from excited ITO substrate to attached metal nanoparticles may be beneficial for dye-MNP composite photoelectrochemical systems to attain cathordic photocurrents by the suppression of unfavorable electron injection from excited dyes into MNPs to some extent. Basic understanding of photoelectrochemical interactions between conducting substrates and metal nanoparticles, as demonstrated a part in this study, will be an essential basics for the designing of MNP-based photoelectrochemical devices.

\section{Acknowledgements}

This research was partially supported by Research Fellowships of Japan Society for the Promotion of Science (JSPS) for young scientists (HAG1004391) and Grant-in-Aid for Scientific Research (No. 19201021) and of the Priority Area (Area code 470) from the Ministry of Education, Culture, Sports, Science and Technology (MEXT) of Japan. 


\section{References}

[1] A. Wood, M. Giersig, P. Mulvaney, Fermi level equilibration in quantum dot-metal nanojunctions, J. Phys. Chem. B 105 (2001) 8810-8815.

[2] V. Subramanian, E. E. Wolf, P. V. Kamat, Green emission to probe photoinduced charging events in $\mathrm{ZnO}-\mathrm{Au}$ nanoparticles. Charge distribution and Fermi-level equilibration, J. Phys. Chem. B 107 (2003) 7479-7485.

[3] V. Subramanian, E. E. Wolf, P. V. Kamat, Catalysis with $\mathrm{TiO}_{2} /$ Gold nanocomposites. effect of metal particle size on the Felmi level equilibration, J. Am. Chem. Soc. 126 (2004) 4943-4950.

[4] T. Hirakawa, P. V. Kamat, Charge separation and catalytic activity of $\mathrm{Ag} @ \mathrm{TiO}_{2}$ core-shell composite clusters under UV-irradiation, J. Am. Chem. Soc. 127 (2005) 3928-3934.

[5] Y. Tian, T. Tatsuma, Mechamisms and applications of plasmon-induced charge separation at $\mathrm{TiO}_{2}$ films loaded with gold nanoparticles, J. Am. Chem. Soc. 127 (2005) 7632-7637.

[6] T. Akiyama, M. Nakada, N. Terasaki, S. Yamada, Photocurrent enhancement in a porphyrin-gold nanoparticle nanostructure assisted by localized plasmon excitation, Chem. Commun. (2006) 395-397.

[7] K. Sugawa, T. Akiyama, H. Kawazumi, S. Yamada, Plasmon-enhansed photocurrent generation from self-assembled monolayers of phthalocyanine by using gold nanoparticle films, Langmuir 25 (2009) 3887-3893.

[8] T. Arakawa, T. Akiyama, S. Yamada, Effect of silver nanoparticles on photoelectrochemical responses of organic dyes, J. Phys. Chem. C 113 (2009) 11830-11835.

[9] M. Lahav, C. H.-Shabtai, J. Wasserman, E. Katz, I. Willner, H. Durr, Y.-Z. Hu, S. H. Bossmann, Photoelectrochemistry with integrated photosensitizer-electron acceptor and Au-nanoparticle arrays, J. Am. Chem. Soc. 122 (2000) 11480-11487.

[10] S. Yamada, T. Tasaki, T. Akiyama, N. Terasaki, S. Nitahara, Gold 
nanoparticle-porphyrin self-assembled multistructures for photoelectric conversion, Thin Solid Films 438-439 (2003) 70-74.

[11] K. Sugawa, T. Kawahara, T. Akiyama, S. Yamada, Structural characterization and photoelectrochemical properties of gold nanoparticle multistructures prepared by layer-by-layer deposition, Jpn. J. Appl. Phys. 48 (2009) 04C132-04C132-5.

[12] T. Arakawa, T. Akiyama, S. Yamada, Structural characterization and photoelectrochemical properties of silver nanoparticle-polyion films, Trans. Mater. Res. Soc. Jpn. 33 (2008) 185-188.

[13] M. D. Malinsky, K. L. Kelly, G. C. Schatz, R. P. Van Duyne, Chain langth dependence and sensing capabilities of the localized surface plasmon resonance of silver nanoparticles chemically modified with alkanethiol self-assembled monolayers, J. Am. Chem. Soc. 123 (2001) 1471-1482.

[14] H. Ahn, A. Chandekar, B. Kang, C. Sung, J. E. Whitten, Electrical conductivity and vapor-sensing properties of $\omega$-(3-thienyl)alkanethiol-protected gold nanoparticle fims, Chem. Mater. 16 (2004) 3274-3278.

[15] M. M. Maye, J. Luo, Y. Lin, M. H. Engelhard, M. Hepel, C.-J. Zhong, X-ray photoelectron spectroscopic study of the activation of molecularly-linked gold nanoparticle catalysts, Langmuir 19 (2003) 125-131.

[16] S. W. Boettcher, S. A. Berg, M. Schierhorn, N. C. Strandwitz, M. C. Lonergan and G. D. Stucky, Ionic ligand mediated electrochemical charging of gold nanoparticle assemblies, Nano Lett. 8 (2008) 3404-3408.

[17] K. Naoi, Y. Ohko, T. Tatsuma, Switchable rewritability of $\mathrm{Ag}-\mathrm{TiO}_{2}$ nanocomposite films with multicolor photochromism, Chem. Commun. (2005) 1288-1290.

[18] W. Wang, S. Efrima, O. Regev, Directing oleate stabilized nanosized silver colloids into organic phases, Langmuir 14 (1998) 602-610.

[19] T. Lvov, K. Ariga, M. Onda, I. Ichinose, T. Kunitake, A careful examination of the adsorption step in the alternate layer-by-layer assembly of linear polyanion and polycation, 
Colloids and Surfaces A 146 (1999) 337-346.

[20] A. J. Capote, A. V. Hamza, N. D. S. Canning, R. J. Madix, The adsorption and reaction of acetonitrile on clean and oxygen covered Ag(110) surfaces, Surf. Sci. 175 (1986) 445-464.

[21] L. M. Doubova, S. Trasatti, S. Valcher, Adsorption of acetonitrile on polycrystalline Ag electrodes: comparison with Hg electrodes, J. Electroanal. Chem. 349 (1993) 187-195.

[22] J, Turkevich, P. C. Stevenson, J. Hiller, A study of the nucleation and growth processes in the synthesis of colloidal gold, Discuss. Faraday. Soc. 11 (1951) 55-75.

[23] F. Matino, L. Persano, V. Arima, D. Pisignano, R. I. R. Blyth, R. Cingolani, R. Rinaldi, Electronic structure of indium-tin-oxide films fabricated by reactive electron-beam deposition, Phys. Rev. B 72 (2005) 085437-085437-6.

[24] J. Szczyrbowski, A. Dietrich, H. Hoffmann, Optical and electrical properties of RF-sputtered indium-tin oxide films, Phys. Stat. Sol. 78 (1983) 243-252.

[25] J. J. McMahon, M. Barry, K. J. Breen, A. K. Radziwon, L. D. Brooks, M. R. Blair, Photocatalysis of the oxygen reduction reaction at adsorbate-covered silver, J. Phys. Chem. C $112(2008)$ 1158-1166.

[26] S. Barazzouk, P. V. Kamat, S. Hotchandani, Photoinduced electron transfer between chlorophyll a and gold nanoparticles, J. Phys. Chem. B 109 (2005) 716-723. 
Figure captions

Fig. 1. (a) SEM image and (d) UV-vis spectrum of ITO/PEI/AgNP.

Fig. 2. Time-dependent change of UV-vis spectra of ITO/PEI/AgNP in air-saturated $\mathrm{H}_{2} \mathrm{O} / \mathrm{MeCN}$ solutions with various volume ratios, unless otherwise noted. Volume rations of $\mathrm{H}_{2} \mathrm{O} / \mathrm{MeCN}$ are: (a) $10 / 0$, (b) $9 / 1$, (c) $7 / 3$, (d) $5 / 5$, (e) $3 / 7$, (f) $0 / 10 \mathrm{v} / \mathrm{v}$, (g) $\mathrm{H}_{2} \mathrm{O} / \mathrm{MeCN}=0 / 10$ with nitrogen bubbling (15 min) prior to measurement, (h) $\mathrm{H}_{2} \mathrm{O} / \mathrm{MeCN}=3 / 7$ with $0.1 \mathrm{mM}$ trisodium citrate.

Fig. 3. Photocurrent action spectra of ITO/PEI/AgNP measured in oxygen-saturated $\mathrm{H}_{2} \mathrm{O} / \mathrm{MeCN}$ electrolyte solutions. Volume rations of $\mathrm{H}_{2} \mathrm{O} / \mathrm{MeCN}$ are: (a) $10 / 0$, (b) $9 / 1$, (c) $7 / 3$, (d) $5 / 5 \mathrm{v} / \mathrm{v}$. (e) Photocurrent action spectrum of ITO/PEI in oxygen-saturated $\mathrm{H}_{2} \mathrm{O} / \mathrm{MeCN}=$ 7/3 electrolyte solution. Applied potential $E=0 \mathrm{~V}$ vs. $\mathrm{Ag} / \mathrm{AgCl}$ (sat. $\mathrm{KCl}$ ). (f) $\mathrm{UV}$-vis spectrum of ITO/PEI/AgNP

Fig. 4. (a) $(\alpha h v)^{N}$ vs. the photon energy $(N=2,1 / 2)$ : solid line, $N=2$; dashed line, $N=1 / 2$, (b) absorption coefficient of ITO substrate.

Fig. 5. Photocurrent action spectra of ITO/PEI/AgNP measured in oxygen-saturated $\mathrm{H}_{2} \mathrm{O} / \mathrm{MeCN}=5 / 5(\mathrm{v} / \mathrm{v})$ solution: (a) without trisodium citrate, (b) with $0.1 \mathrm{mM}$ trisodium citrate, (c) ODT-modified AgNP, without trisodium citrate. Applied potential $E=0 \mathrm{~V}$ vs. $\mathrm{Ag} / \mathrm{AgCl}$ (sat. $\mathrm{KCl})$. 

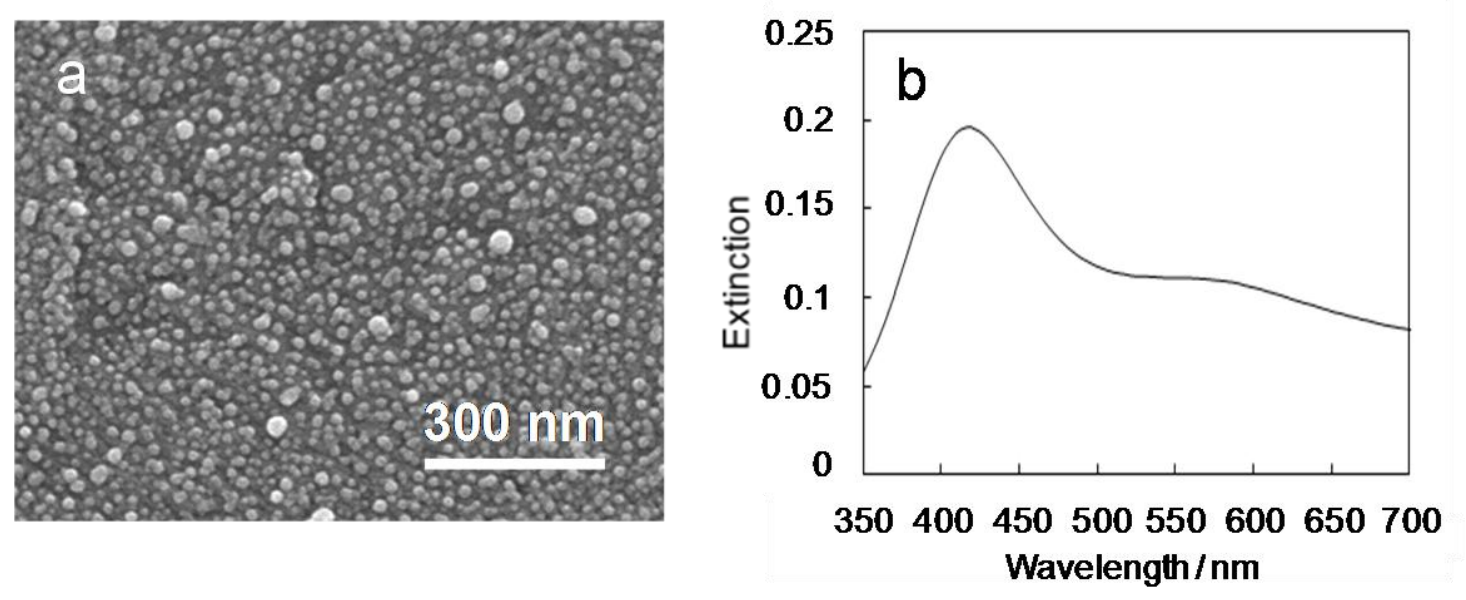

Fig. 1. Matsuoka et al. 

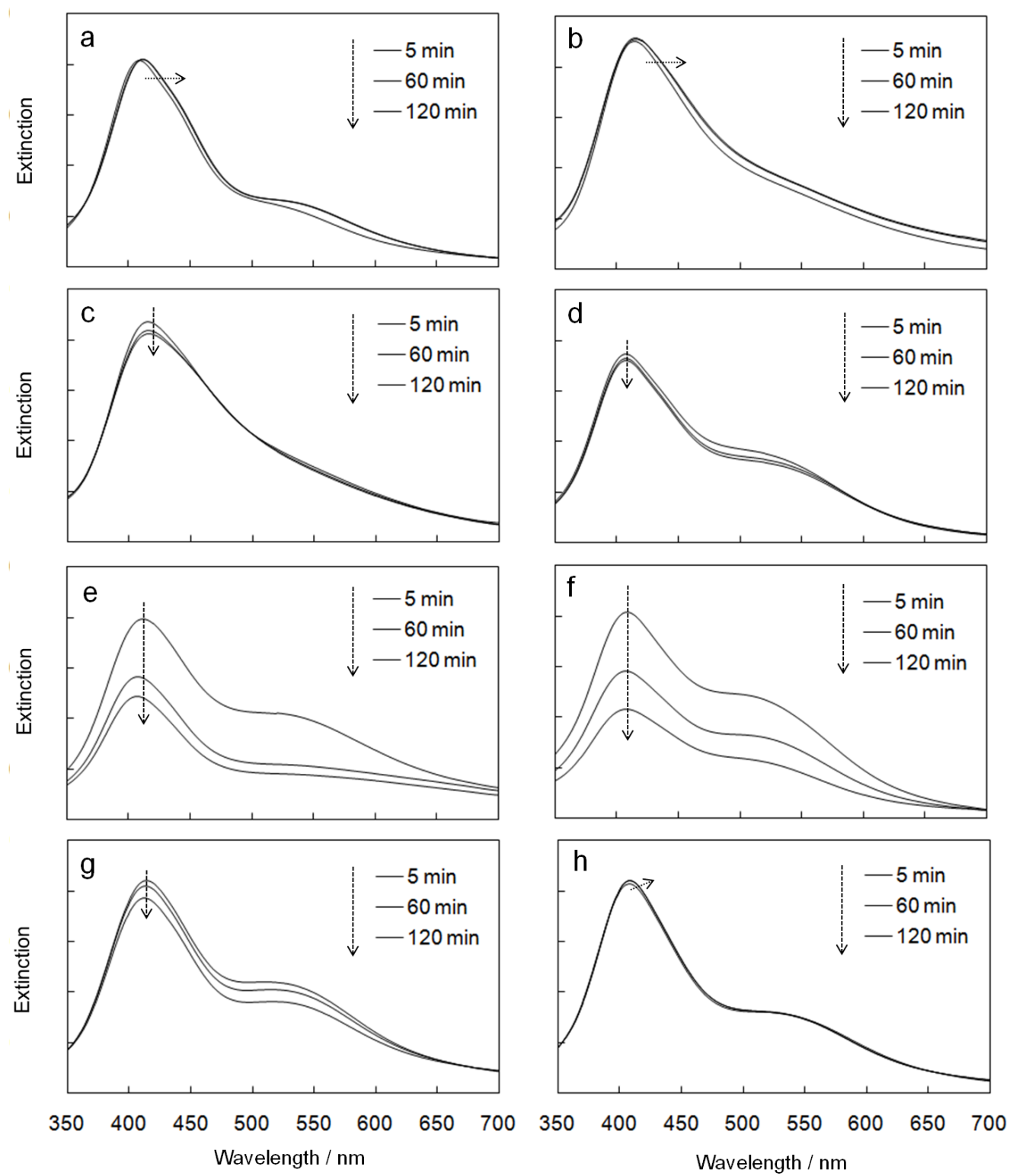

Fig. 2. Matsuoka et al. 


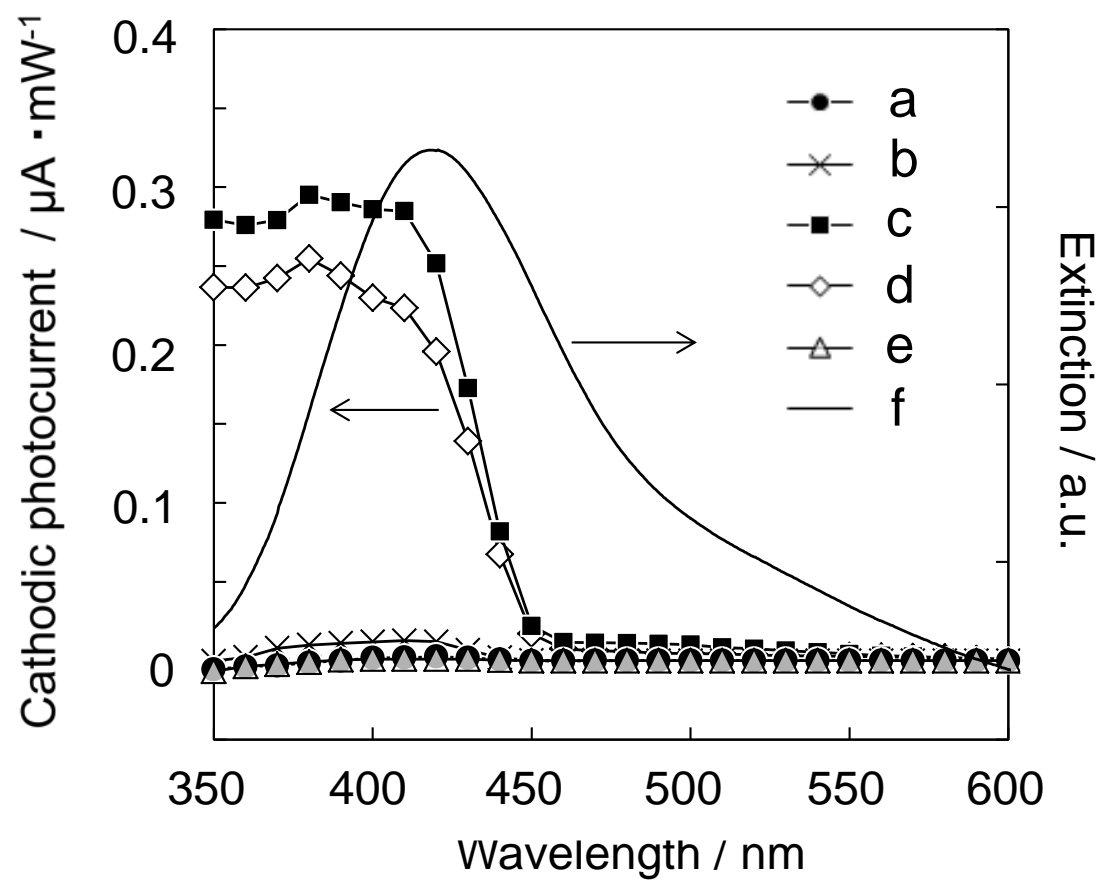

Fig. 3. Matsuoka et al. 

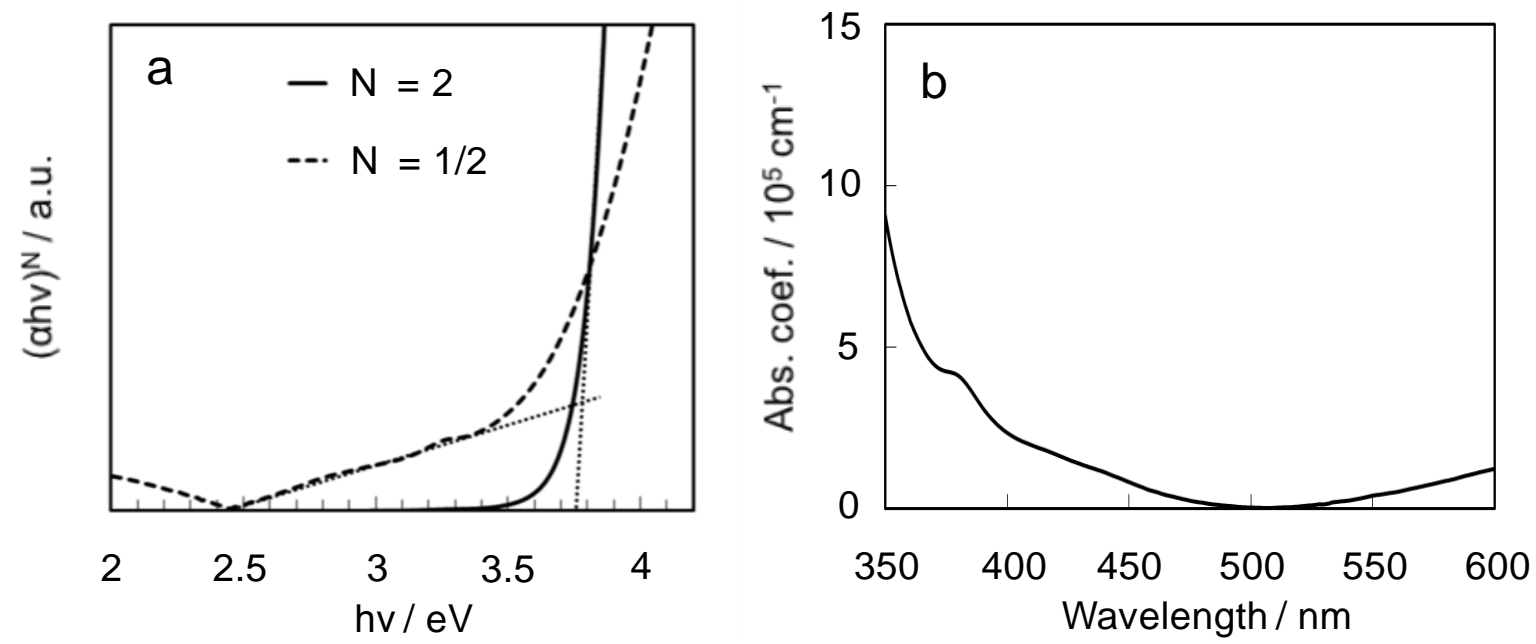

Fig. 4. Matsuoka et al. 


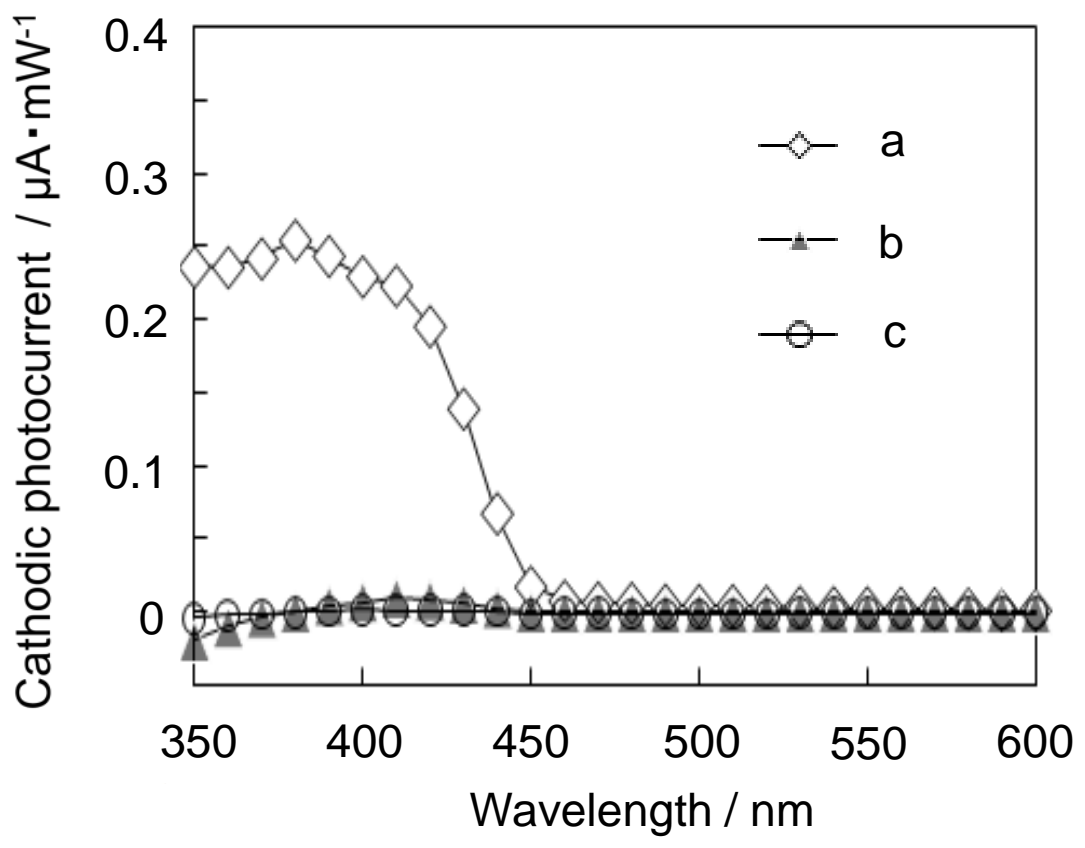

Fig. 5. Matsuoka et al. 